\title{
Macroscopic diffusivity in concrete determined by computational homogenization
}

\author{
Filip Nilenius ${ }^{1}$, Fredrik Larsson ${ }^{2}$, Karin Lundgren ${ }^{1}$, and \\ Kenneth Runesson ${ }^{2}$ \\ ${ }^{1}$ Department of Civil and Environmental Engineering, Chalmers \\ University of Technology \\ ${ }^{2}$ Department of Applied Mechanics, Chalmers University of \\ Technology
}

May 31, 2012

\begin{abstract}
Effective moisture and chloride ion diffusivity coefficients for concrete are determined by computational homogenization, where concrete is modelled on the mesoscale as a heterogenous three-phase composite material. By imposing moisture and chloride ion gradients on a Representative Volume Element (RVE), effective macroscale properties are obtained through finite element analysis. A parametric study of the effects of the aggregate content was carried out. The numerical results correspond well with an estimate of the Hashin-Shtrikman type, available in the literature. The computational homogenization strategy proposed here also includes the Interfacial Transition Zone (ITZ), and its influence on the effective diffusivity coefficients is assessed.
\end{abstract}




\section{Introduction}

For concrete structures, deteriorating mechanisms are of great concern for both safety and economy reasons. One such important mechanism is that of chloride ion ingress. Chloride ions ${ }^{1}$ are harmful for concrete structures since they can initiate corrosion of embedded reinforcement bars at a certain level of concentration, which subsequently may lead to fracture and structural collapse, see e.g. [18, 27] for the mechanical behavior of deteriorated concrete structures. The porosity of the cement paste allows for diffusion of the chloride ions; however, since the chloride ions are dissolved in water ${ }^{2}$, the mass transfer of chloride ions will also be driven by (and coupled to) the moisture transport. On the other token, a change of ion concentration will affect the rate of moisture transport; hence, the mass transport of chloride ions and moisture is a truly coupled problem. In conclusion, it is important to be able to simulate and predict ingress of these harmful ions together with moisture transfer, in a realistic way, to foresee weakening of concrete structures due to chloride initiated corrosion.

The aim of the work reported here is to investigate the effects of ballast and the Interfacial Transition Zone (ITZ) on the coupled moisture and chloride ion diffusion in concrete. To this end, concrete on the mesoscale is considered as a composite material consisting of ballast particles embedded in the cement paste matrix. The described coupled diffusion problem was modeled in [21] for homogenous concrete, whereby the effect of ballast was introduced via an estimate of the effective diffusion of the Hashin-Shtrikman type [11]. It may be noted that the same type of estimate was used in [3] for estimating thermal conductivity in composite materials with spherical inclusions. In the present article, however, the effect of ballast is quantified from direct homogenization of the response of the mesostructure. By doing so, the effect can be better analyzed and controlled. Moreover, the geometric representation of ballast enables the ITZ between the ballast and cement paste to be included in the model as a third phase. The effect of ITZ has previously been investigated by, e.g. $[4,13,25]$.

Since the material properties are highly nonlinear, the homogenization must be carried out on a Representative Volume Element (RVE) of the

\footnotetext{
${ }^{1}$ Only the movement of dissolved, or free, chloride ions are of concern, as opposed to the bound ones.

${ }^{2}$ The chloride ions are dissolved only in the liquid phase (and not in the vapor phase); however, this fact is of no consequence for the modelling of moisture transport.
} 
concrete. Multiscale methods, including homogenization, for transport phenomena in concrete, and porous media, have been adopted by several authors, cf. [19, 16, 10, 17, 15]. Herein, we shall adopt so-called "first order homogenization", as it is only the values of the macroscale solution of the scalar fields (moisture and chloride ions) and their spatial gradients that are used as 'loading' for the mesoscale modelling, cf. [6, 14, 26] and [12] where the first order homogenization approach was included in a $\mathrm{FE}^{2}$-scheme.

The paper is organized as follows: In Section 2, the procedure for numerical generation of an RVE is described together with the constitutive model used for the cement paste. Section 3 describes the computational homogenization of the transport problem under the restriction to stationary conditions but for general nonlinear response. The specialization to linearized macroscopic response is described in Section 4, and the corresponding solution strategy for determining the diffusivity tensors based on the pertinent sensitivity problem is outlined. Numerical results are presented in Section 5, where particular emphasis is placed on the influence of ballast on the diffusivity, both including and excluding ITZ. Finally, Section 6 contains conclusions along with a suggestion for future work.

\section{Mesoscale modelling of concrete}

\subsection{Characterization of the mesoscale structure - SVE generation}

Concrete is considered as a three-phase composite material, consisting of the cement paste, ballast and ITZ. Each phase is assumed to be homogenous. The ITZ is introduced as a highly diffusive interface "material" along the perifery of each ballast particle, cf. Section 5.3.

At the core of homogenization analysis is the generation of an SVE (= Statistical Volume Element) of the mesoscale structure of concrete. An SVE is a realization of a stochastic field and is generated from a given statistical representation of the subscale features; however, its size can be considerably smaller than the RVE which must be sufficiently large that any realization gives essentially the same response. For a discussion of the requirements on the RVE and SVE's, see [28]. Various strategies for the generation of SVE's have been devised in the literature. Voronoi polygonization was used in $[20,8,22]$ to numerically generate a 2D-structure. This idea was extended 
to 3D mesostructures in [2] in the context of fracture analysis of concrete. CT-scanning technique was used in [10] to generate 3D micro-structures of cement paste.

In this paper, we have developed a strategy for the numerical generation of 2D structures of SVE's with the subsequent FE-meshing. The main control parameters of the algorithm are the area fraction of ballast, denoted $n_{\mathrm{b}}$, and the sieve curve of ballast. At the outset, each ballast particle is an octagon with the radius $r$, see Figure 1a. The randomness in shape of the ballast is simulated by adding a random variation to the radius of each corner point in the octagon, see Figure 1b. The radius variation lies in the closed interval $[-0.25 r, 0.25 r]$.

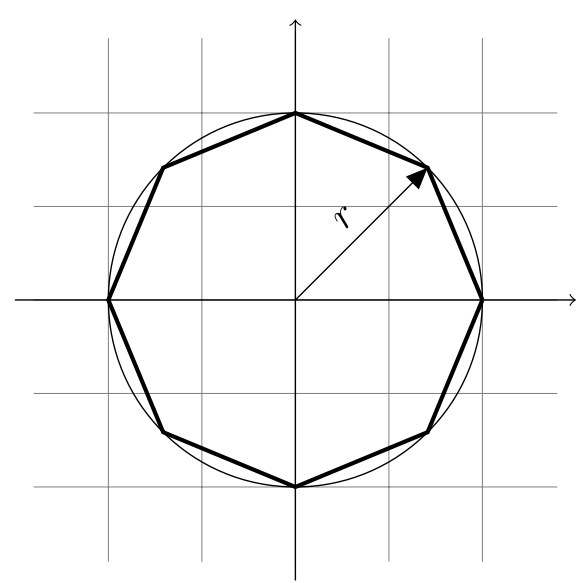

(a) Octagon bounded by a circle with radius $r$.

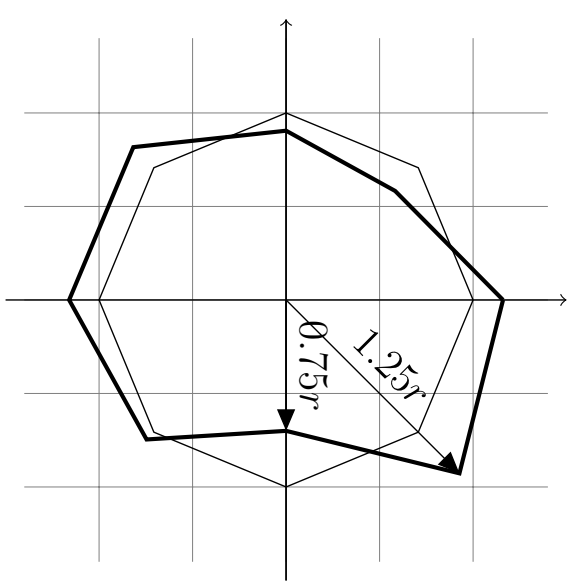

(b) Applied random variations in $r$ to the corner points of the octagon.

Figure 1: Ballast particle modeled as randomly perturbed octagon.

For a given subscale domain $\Omega_{\square}$ in $2 \mathrm{D}$, representing the SVE, the algorithm has the following principal structure:

- while the area fraction of ballast inside $\Omega_{\square}<n_{\mathrm{b}}$

- Generate a new ballast particle with radius according to given sieve curve

- Include random variation in its radius 
- Place the new ballast particle at a random point in $\Omega_{\square}$

- if the center of the new ballast particle is inside an already existing one

* Remove the new ballast particle

- end

- if the new ballast particle overlaps an already existing one

* Scale the ballast down stepwise until overlap is avoided

- end

- add area of the new ballast particle to the accumulated ballast area

- end

Remark 1 An experimentally obtained sieve curve (from 3D particles) would have to be re-scaled when used in a $2 D$ model since the sieve curve data need to be mapped from a volume to a surface. However, such re-scaling is beyond the scope of this article and the sieve curve data used in the numerical examples are assumed to be valid for a $2 D$ model.

The algorithm guarantees that any overlap of ballast is avoided, and new particles are added to the SVE until the desired ballast area fraction, $n_{\mathrm{b}}$, is reached. However, a consequence of avoiding overlaps of the ballast particles is that the specified sieve curve is violated. The final amount of large ballast particles will be lower than specified, while small ballast particles will be over represented. This violation effect can be seen in Figure 2. It is noteworthy that the ballast content specified within the SVE is unaffected by this shift in sieve curve. Examples of generated SVEs with three different ballast fractions are shown in Figure 3. 


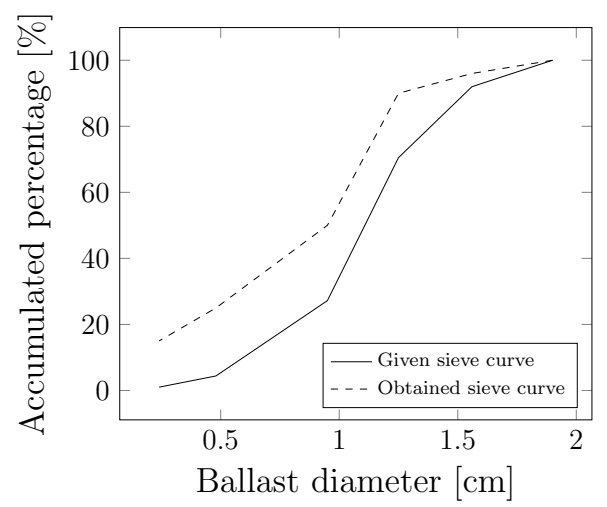

Figure 2: Sieve curves for the model.

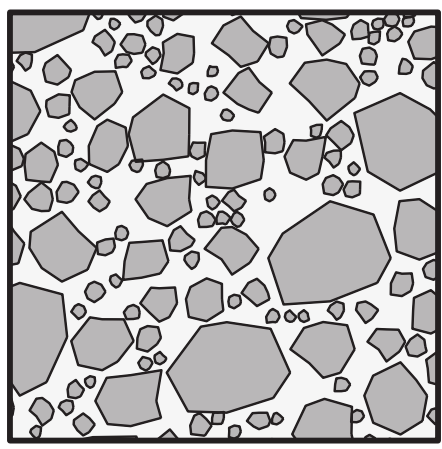

(a) $n_{b} \approx 60 \%$

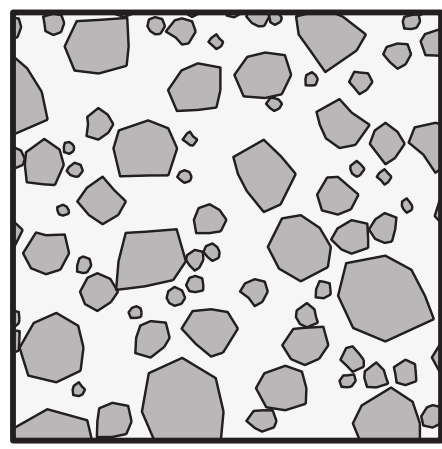

(b) $n_{b} \approx 50 \%$

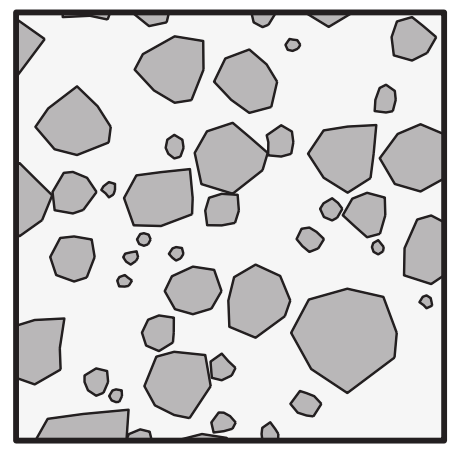

(c) $n_{b} \approx 40 \%$

Figure 3: Examples of generated SVEs with different ballast area fractions.

\subsection{Balance equations - Moisture and chloride trans- port}

In this paper we consider only stationary transport conditions, in which case the pertinent balance equations of mass conservation on the "fine scale" are simply

$$
\begin{array}{ll}
\boldsymbol{\nabla} \cdot \boldsymbol{J}_{\phi}=0 & \forall \boldsymbol{x} \in \Omega \\
\boldsymbol{\nabla} \cdot \boldsymbol{J}_{\mathrm{c}}=0 & \forall \boldsymbol{x} \in \Omega,
\end{array}
$$


where $\boldsymbol{J}_{\phi}$ and $\boldsymbol{J}_{\mathrm{c}}$ are the mass flux (vectors) of moisture and chloride ions, respectively. Both are assumed, at the outset, to depend in a quite general fashion on the non-dimensional fields $\phi$ and $c$ and on their spatial gradients, $\boldsymbol{\nabla} \phi$ and $\boldsymbol{\nabla} c$, where $\phi$ is the relative humidity in the cement paste pores and where $c$ is the chloride ion concentration, defined here as the ratio of chloride ion mass to cement paste mass in a unit volume. Hence, Eqs. (1) and (2) represent coupled equations in the primary fields $\phi$ and $c$.

The pertinent boundary conditions are (in standard fashion) either of the Dirichlet type (prescribed values of $\Gamma_{\mathrm{D}}^{(\phi)}$ and $\Gamma_{\mathrm{D}}^{(c)}$, respectively) or of the Neumann type (prescribed values of boundary fluxes on $\Gamma_{\mathrm{N}}^{(\phi)}$ and $\Gamma_{\mathrm{N}}^{(c)}$, respectively), whereby $\Gamma=\Gamma_{\mathrm{D}}^{(\phi)} \cup \Gamma_{\mathrm{N}}^{(\phi)}=\Gamma_{\mathrm{D}}^{(c)} \bigcup \Gamma_{\mathrm{N}}^{(c)}$. The corresponding weak format then reads

$$
\begin{aligned}
\int_{\Omega} \nabla \delta \phi \cdot \boldsymbol{J}_{\phi} \mathrm{d} \Omega & =\int_{\Gamma_{\mathrm{N}}^{(\phi)}} \delta \phi J_{\phi, n} \mathrm{~d} \Gamma \\
\int_{\Omega} \boldsymbol{\nabla} \delta c \cdot \boldsymbol{J}_{\mathrm{c}} \mathrm{d} \Omega & =\int_{\Gamma_{\mathrm{N}}^{(c)}} \delta c J_{\mathrm{c}, n} \mathrm{~d} \Gamma
\end{aligned}
$$

for suitable test functions $\delta \phi$ and $\delta c$ that are sufficiently regular and satisfy homogeneous boundary conditions on $\Gamma_{\mathrm{D}}^{(\phi)}$ and $\Gamma_{\mathrm{D}}^{(c)}$, respectively. The only

"loading" terms here are the prescribed values of outward fluxes $J_{\phi, n} \stackrel{\text { def }}{=} \boldsymbol{J}_{\phi} \cdot \boldsymbol{n}$ and $J_{\mathrm{c}, n} \stackrel{\text { def }}{=} \boldsymbol{J}_{\mathrm{c}} \cdot \boldsymbol{n}$, respectively.

\subsection{Constitutive relations for the mesoscale materials}

\subsubsection{Preliminaries}

Although the transport of moisture and chloride ions is partly of the convective type, they will be modeled as diffusion processes, whereby concentration gradients of moisture and chloride ions are the driving forces on the fine scale. In principle, such transport takes place in all constituents; however, for all practical purposes it can be assumed that the ballast particles are completely impervious.

\subsubsection{Diffusion properties of the cement paste}

Experimental results found in [1] indicate that the transport of moisture and chloride ions are cross-coupled in the pure cement matrix. Based on these 
results, a "quasi-linear" constitutive model was proposed by them as follows:

$$
\begin{aligned}
\boldsymbol{J}_{\phi}(\phi, c ; \boldsymbol{\nabla} \phi, \boldsymbol{\nabla} c) & =-D_{\phi}(\phi) \boldsymbol{\nabla} \phi-\varepsilon_{\mathrm{c}} D_{\mathrm{c}}(\phi, c) \boldsymbol{\nabla} c, \\
\boldsymbol{J}_{\mathrm{c}}(\phi, c ; \boldsymbol{\nabla} \phi, \boldsymbol{\nabla} c) & =-\varepsilon_{\phi} D_{\phi}(\phi) \boldsymbol{\nabla} \phi-D_{\mathrm{c}}(\phi, c) \boldsymbol{\nabla} c,
\end{aligned}
$$

where it is assumed from the outset that the intrinsic diffusion properties (for the pure cement paste), represented by the diffusion coefficients $D_{\phi}>0$ and $D_{\mathrm{c}}>0$, are isotropic. Furthermore, $\varepsilon_{\phi}$ and $\varepsilon_{\mathrm{c}}$ are coupling parameters, which are taken as constants according to [21] $\left(\varepsilon_{\phi}=0.028 \text { and } \varepsilon_{\mathrm{c}}=0.2\right)^{3}$. The expressions Eqs. (5) and (6) can be seen as modifications of Fick's classical law of diffusion.

The pertinent expressions for the state-dependent diffusion coefficients adopted here were proposed, together with values of the parameters $\alpha_{h}, \beta_{h}$, $\gamma_{h}, f_{1}, f_{2}, f_{3}, f_{4}$ and $f_{5}$, in $[24,23]$. They are as follows:

$$
\begin{array}{rlrl}
D_{\phi}(\phi) & =\alpha_{h}+\beta_{h}\left[1-2^{\left.-10^{\gamma_{h}[\phi-1]}\right]}\right. & & {\left[\mathrm{cm}^{2} / \text { day }\right],} \\
D_{\mathrm{c}}(\phi, c) & =f_{1}\left(t_{0}\right) f_{2}\left(n_{\mathrm{b}}\right) f_{3}(\phi) f_{4}(T) f_{5}(c) & {\left[\mathrm{cm}^{2} / \text { day }\right],}
\end{array}
$$

where $t_{0}$ is the curing time and $T$ is the temperature. How $D_{\phi}$ and $D_{\text {c }}$ depend on their respective arguments is illustrated in Figures $4 \mathrm{a}$ and $4 \mathrm{~b}$.

\footnotetext{
${ }^{3}$ We shall require the "compound diffusivity" to be positive definite in the sense that $\boldsymbol{J}_{\phi} \cdot \boldsymbol{\nabla} \phi+\boldsymbol{J}_{\mathrm{c}} \cdot \boldsymbol{\nabla} c<0$ for any $\boldsymbol{\nabla} \phi \neq \mathbf{0}$ and $\boldsymbol{\nabla} c \neq \mathbf{0}$. This is equivalent to the requirement $1-\varepsilon_{\phi} \varepsilon_{\mathrm{c}}>0$, which is, obviously, satisfied for the chosen values of $\varepsilon_{\phi}$ and $\varepsilon_{\mathrm{c}}$.
} 


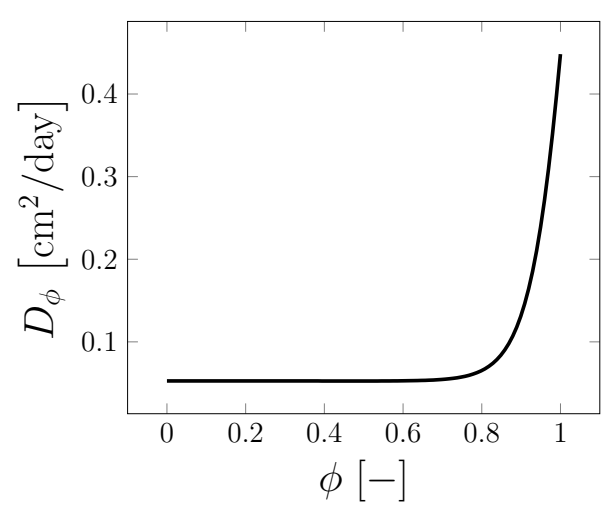

(a)

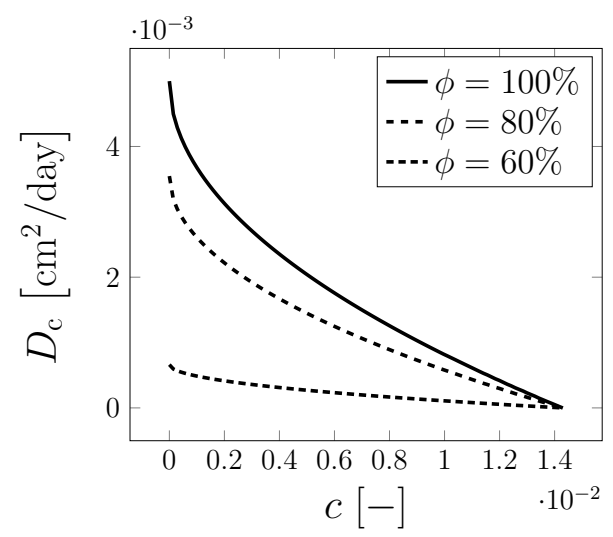

(b)

Figure 4: State-dependent diffusion coefficients $D_{\phi}(\phi)$ and $D_{c}(\phi, c)$.

\subsubsection{Diffusion properties of the ITZ}

For the ITZ, we assume a constitutive relation with the same structure as that of the cement paste, described in the previous subsection. However, the diffusion coefficients for ITZ do not represent a homogeneous material but rather the net effect of pores and microcracks within a thin zone of thickness $h$. We shall treat ITZ as a 1D continuum material "superimposed" on the mesoscale domain, implying that additional mass transport in the ITZ is allowed only in the plane of the ballast/cement interface, which is denoted by $\|$, see Figure 5. Consequently, the constitutive relations take the form

$$
\begin{aligned}
& \boldsymbol{J}_{\phi, \|}(\boldsymbol{\nabla} \phi, \boldsymbol{\nabla} c)=-D_{\phi, \mathrm{ITZ}} \boldsymbol{\nabla}_{\|} \phi-\varepsilon_{\mathrm{c}} D_{\mathrm{c}, \mathrm{ITZ}} \boldsymbol{\nabla}_{\|} c, \\
& \boldsymbol{J}_{\mathrm{c}, \|}(\boldsymbol{\nabla} \phi, \boldsymbol{\nabla} c)=-\varepsilon_{\phi} D_{\phi, \mathrm{ITZ}} \boldsymbol{\nabla}_{\|} \phi-D_{\mathrm{c}, \mathrm{ITZ}} \boldsymbol{\nabla}_{\|} c,
\end{aligned}
$$

where $\boldsymbol{\nabla}_{\|}=\boldsymbol{\nabla} \cdot\left[\boldsymbol{I}-\boldsymbol{e}_{\perp} \otimes \boldsymbol{e}_{\perp}\right]$. Here, we introduced $\boldsymbol{J}_{\phi, \|}$ and $\boldsymbol{J}_{\mathrm{c}, \|}$ as the mass flux vectors along the ITZ; hence, the orthogonal components are zero by construction.

FE-discretization of the ITZ gives the following typical interface element relation

$$
\left[\begin{array}{l}
\underline{J}_{\phi}^{e} \\
\underline{J}_{\mathrm{c}}^{e}
\end{array}\right]=-\left[\begin{array}{ll}
\underline{K}_{\phi \phi}^{e} & \underline{K}_{\phi \mathrm{c}}^{e} \\
\underline{K}_{\mathrm{c} \phi}^{e} & \underline{K}_{\mathrm{cc}}^{e}
\end{array}\right]\left[\begin{array}{c}
\phi^{e} \\
\underline{c}^{e}
\end{array}\right],
$$


where $\phi^{e}$ and $\underline{c}^{e}$ are the nodal values of $\phi$ and $c$, respectively. Using piecewise linear interpoation of $\phi$ and $c$, the submatrices, representing diffusion resistance, take the simple explicit forms

$$
\begin{gathered}
\underline{K}_{\phi \phi}^{e}=\frac{D_{\phi, \mathrm{ITZ}} A_{\mathrm{ITZ}}}{L_{e}}\left[\begin{array}{rr}
1 & -1 \\
-1 & 1
\end{array}\right], \\
\underline{K}_{\phi \mathrm{c}}^{e}=\varepsilon_{\mathrm{c}} \frac{D_{\mathrm{c}, \mathrm{ITZ}} A_{\mathrm{ITZ}}}{L_{e}}\left[\begin{array}{rr}
1 & -1 \\
-1 & 1
\end{array}\right], \\
\underline{K}_{\mathrm{c} \phi}^{e}=\varepsilon_{\phi} \frac{D_{\phi, \mathrm{ITZ}} A_{\mathrm{ITZ}}}{L_{e}}\left[\begin{array}{rr}
1 & -1 \\
-1 & 1
\end{array}\right], \\
\underline{K}_{\mathrm{cc}}^{e}=\frac{D_{\mathrm{c}, \mathrm{ITZ}} A_{\mathrm{ITZ}}}{L_{e}}\left[\begin{array}{rr}
1 & -1 \\
-1 & 1
\end{array}\right],
\end{gathered}
$$

where $A_{\text {ITZ }}$ is the cross-sectional area of the ITZ layer with unit thickness, and $L_{e}$ is the length of the ITZ element. The ITZ was assigned a width $h=60 \mu \mathrm{m}^{2}$ in the FE-model, which yields $A_{\mathrm{ITZ}}=1 \times 60\left[\mu \mathrm{m}^{2}\right]$. Hence, it is only the products $D_{\phi, \mathrm{ITZ}} A_{\mathrm{ITZ}}$ and $D_{\mathrm{c}, \mathrm{ITZ}} A_{\mathrm{ITZ}}$ that are determined from experimental data via an inverse analysis, cf Section 5.3.

Remark 2 It is noted that the FE-discretized fields $\phi$ and $c$ are continuous across the interface elements.

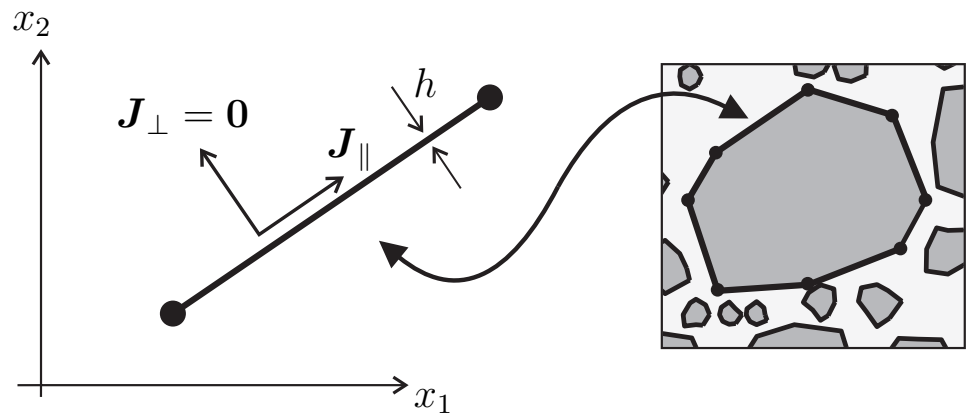

Figure 5: ITZ interface elements along the perifery of each ballast particle.

The approach of including ITZ as interface elements for mesoscale modelling of concrete was also employed in [22] to model water flow, and in [7] to model the increase of concrete diffusivity due to cracking, in the context of fracture analysis. 


\section{First order homogenization - General non- linear response}

\subsection{Macroscale problem}

Homogenization infers that the integrands of the volume integrals in Eqs. (3) and (4) are replaced by space-averages on "representative volume elements", i.e.

$$
\int_{\Omega} \boldsymbol{\nabla} \delta \phi \cdot \boldsymbol{J}_{\phi} \mathrm{d} \Omega \longrightarrow \int_{\Omega}\left\langle\boldsymbol{\nabla} \delta \phi \cdot \boldsymbol{J}_{\phi}\right\rangle_{\square} \mathrm{d} \Omega
$$

where $\langle\bullet\rangle_{\square}$ denotes the volume average

$$
\langle\bullet\rangle_{\square} \stackrel{\text { def }}{=} \frac{1}{\left|\Omega_{\square}\right|} \int_{\Omega_{\square}} \bullet \mathrm{d} \Omega_{\square},
$$

on the RVE that occupies the domain $\Omega_{\square}$ centered at the macroscale position $\overline{\boldsymbol{x}} \in \Omega$; hence, $\langle\boldsymbol{x}-\overline{\boldsymbol{x}}\rangle_{\square}=0$.

In standard fashion, we assume first order homogenization defined by the split of a typical field, say $\phi$, within $\Omega_{\square}$ into the macroscale and fluctuation parts $^{4}$ as follows:

$$
\phi(\boldsymbol{x} ; \overline{\boldsymbol{x}})=\phi^{M}(\boldsymbol{x} ; \overline{\boldsymbol{x}})+\phi^{s}(\boldsymbol{x}) .
$$

The macroscale part varies linearly as

$$
\phi^{M}(\boldsymbol{x} ; \overline{\boldsymbol{x}})=\bar{\phi}(\overline{\boldsymbol{x}})+\overline{\boldsymbol{g}}_{\phi}(\overline{\boldsymbol{x}}) \cdot[\boldsymbol{x}-\overline{\boldsymbol{x}}] \quad \forall \boldsymbol{x} \in \Omega_{\square},
$$

with $\overline{\boldsymbol{g}}_{\phi} \stackrel{\text { def }}{=} \bar{\nabla} \bar{\phi}$. Hence, we obtain $\boldsymbol{\nabla} \phi^{M}(\overline{\boldsymbol{x}} ; \overline{\boldsymbol{x}})=\overline{\boldsymbol{g}}_{\phi}(\overline{\boldsymbol{x}})$ constant within $\Omega_{\square}$. We then obtain for any macroscale point $\overline{\boldsymbol{x}} \in \Omega$ the identity

$$
\left\langle\nabla \delta \phi \cdot \boldsymbol{J}_{\phi}\right\rangle_{\square}=\delta \overline{\boldsymbol{g}}_{\phi} \cdot \overline{\boldsymbol{J}}_{\phi} \quad \text { with } \quad \overline{\boldsymbol{J}}_{\phi}=\left\langle\boldsymbol{J}_{\phi}\right\rangle_{\square} .
$$

Remark 3 The macroscale flux vectors depend in an implicit fashion on the macroscale fields $\bar{\phi}, \bar{c}$ and their spatial gradients denoted $\overline{\boldsymbol{g}}_{\phi}, \overline{\boldsymbol{g}}_{c}$; hence, we write $\overline{\boldsymbol{J}}_{\phi}=\overline{\boldsymbol{J}}_{\phi}\left\{\bar{\phi}, \bar{c} ; \overline{\boldsymbol{g}}_{\phi}, \overline{\boldsymbol{g}}_{c}\right\}$ and $\overline{\boldsymbol{J}}_{c}=\overline{\boldsymbol{J}}_{c}\left\{\bar{\phi}, \bar{c} ; \overline{\boldsymbol{g}}_{\phi}, \overline{\boldsymbol{g}}_{c}\right\}$.

\footnotetext{
${ }^{4}$ Superscripts $M$ denotes macroscale, whereas $s$ denotes subscale.
} 
Now, assuming that the prescribed boundary fluxes are sufficiently smooth, we may ignore fluctuations on the Neumann parts of the boundary $\Gamma$; hence, the macroscale problem can be written as follows:

$$
\begin{aligned}
\int_{\Omega} \delta \overline{\boldsymbol{g}}_{\phi} \cdot \overline{\boldsymbol{J}}_{\phi} \mathrm{d} \Omega & =\int_{\Gamma_{\mathrm{N}}^{(\phi)}} \delta \bar{\phi} J_{\phi, \mathrm{n}} \mathrm{d} \Gamma, \\
\int_{\Omega} \delta \overline{\boldsymbol{g}}_{\mathrm{c}} \cdot \overline{\boldsymbol{J}}_{\mathrm{c}} \mathrm{d} \Omega & =\int_{\Gamma_{\mathrm{N}}^{(c)}} \delta \bar{c} J_{\mathrm{c}, \mathrm{n}} \mathrm{d} \Gamma,
\end{aligned}
$$

which must hold for sufficiently regular $\delta \bar{\phi}$ and $\delta \bar{c}$ that satisfy homogeneous boundary conditions on $\Gamma_{\mathrm{D}}^{(\phi)}$ and $\Gamma_{\mathrm{D}}^{(c)}$, respectively.

\subsection{RVE problem with Dirichlet boundary conditions}

In order to compute the homogenized flux quantities $\overline{\boldsymbol{J}}_{\phi}$ and $\overline{\boldsymbol{J}}_{\phi}$, it is necessary to compute the fluctuation fields $\phi^{s}$ and $c^{s}$ as well as their spatial gradients on the subscale: $\boldsymbol{g}_{\phi}^{s} \stackrel{\text { def }}{=} \boldsymbol{\nabla} \phi^{s}$ and $\boldsymbol{g}_{\mathrm{c}}^{s} \stackrel{\text { def }}{=} \boldsymbol{\nabla} c^{s}$. This is done on each RVE with given prolongation conditions. Here, we choose (for the sake of simplicity) Dirichlet boundary conditions on both fields, i. e. $\phi^{s}=c^{s}=0$ on $\Gamma_{\square}$, whereby the pertinent RVE-problem becomes: For given values of the macroscale variables $\bar{\phi}, \bar{c}$ and $\overline{\boldsymbol{g}}_{\phi}, \overline{\boldsymbol{g}}_{\mathrm{c}}$, compute the subscale fields $\phi^{s}, c^{s}$ that satisfy the coupled system

$$
\begin{aligned}
\left\langle\boldsymbol{\nabla}\left(\delta \phi^{s}\right) \cdot \boldsymbol{J}_{\phi}\right\rangle_{\square} & =0, \\
\left\langle\boldsymbol{\nabla}\left(\delta c^{s}\right) \cdot \boldsymbol{J}_{\mathrm{c}}\right\rangle_{\square} & =0,
\end{aligned}
$$

for all possible $\delta \phi^{s}, \delta c^{s}$ that are sufficiently regular and which vanish on $\Gamma_{\square}$. We note that the macroscale prolongations $\phi^{M}, c^{M}$ are completely defined (varies linearly) on $\Omega_{\square}$.

In order to solve the (nonlinear) system Eqs. (23) and (24), it is convenient to use Newton iterations, whereby linearization of the constitutive relations for the fluxes gives

$$
\begin{aligned}
\mathrm{d} \boldsymbol{J}_{\phi} & =-\boldsymbol{D}_{\phi \phi} \cdot \boldsymbol{\nabla}(\mathrm{d} \phi)-\boldsymbol{Y}_{\phi \phi} \mathrm{d} \phi-\boldsymbol{D}_{\phi c} \cdot \boldsymbol{\nabla}(\mathrm{d} c)-\boldsymbol{Y}_{\phi c} \mathrm{~d} c \\
\mathrm{~d} \boldsymbol{J}_{\mathrm{c}} & =-\boldsymbol{D}_{\mathrm{c} \phi} \cdot \boldsymbol{\nabla}(\mathrm{d} \phi)-\boldsymbol{Y}_{\mathrm{c} \phi} \mathrm{d} \phi-\boldsymbol{D}_{\mathrm{cc}} \cdot \boldsymbol{\nabla}(\mathrm{d} c)-\boldsymbol{Y}_{\mathrm{cc}} \mathrm{d} c
\end{aligned}
$$


whereby a comparison with the explicit choice of constitutive relations in Eqs. (5) and (6) shows that the tangent tensors in Eqs. (25) and (26) become

$$
\begin{array}{llll}
\boldsymbol{D}_{\phi \phi}=D_{\phi} \boldsymbol{I} & \boldsymbol{Y}_{\phi \phi}=\left(D_{\phi}\right)_{\phi}^{\prime} \boldsymbol{\nabla} \phi & \boldsymbol{D}_{\phi \mathrm{c}}=\varepsilon_{\mathrm{c}} D_{\mathrm{c}} \boldsymbol{I} & \boldsymbol{Y}_{\phi \mathrm{c}}=\varepsilon_{\mathrm{c}}\left(D_{\mathrm{c}}\right)_{\mathrm{c}}^{\prime} \boldsymbol{\nabla} c \\
\boldsymbol{D}_{\mathrm{c} \phi}=\varepsilon_{\phi} D_{\phi} \boldsymbol{I} & \boldsymbol{Y}_{\mathrm{c} \phi}=\varepsilon_{\phi}\left(D_{\phi}\right)_{\phi}^{\prime} \boldsymbol{\nabla} \phi & \boldsymbol{D}_{\mathrm{cc}}=D_{\mathrm{c}} \boldsymbol{I} & \boldsymbol{Y}_{\mathrm{cc}}=\left(D_{\mathrm{c}}\right)_{\mathrm{c}}^{\prime} \boldsymbol{\nabla} c .
\end{array}
$$

A Newton step is then defined as

$$
\phi^{s(k+1)}=\phi^{s(k)}+\Delta \phi^{s}, \quad c^{s(k+1)}=c^{s(k)}+\Delta c^{s},
$$

where the iterative increments $\Delta \phi^{s}, \Delta c^{s}$ are solutions of the system

$$
\begin{aligned}
& \left\langle\boldsymbol{\nabla} \delta \phi^{s} \cdot\left[\boldsymbol{D}_{\phi \phi} \cdot \boldsymbol{\nabla}\left(\Delta \phi^{s}\right)+\boldsymbol{Y}_{\phi \phi} \Delta \phi^{s}\right]\right\rangle_{\square} \\
& +\left\langle\boldsymbol{\nabla} \delta \phi^{s} \cdot\left[\boldsymbol{D}_{\phi \mathrm{c}} \cdot \boldsymbol{\nabla}\left(\Delta c^{s}\right)+\boldsymbol{Y}_{\phi \mathrm{c}} \Delta c^{s}\right]\right\rangle_{\square}=\left\langle\boldsymbol{\nabla} \delta \phi^{s} \cdot \boldsymbol{J}_{\phi}^{(k)}\right\rangle_{\square}, \\
& \left\langle\boldsymbol{\nabla} \delta c^{s} \cdot\left[\boldsymbol{D}_{\mathrm{c} \phi} \cdot \boldsymbol{\nabla}\left(\Delta \phi^{s}\right)+\boldsymbol{Y}_{\mathrm{c} \phi} \Delta \phi^{s}\right]\right\rangle_{\square} \\
& +\left\langle\boldsymbol{\nabla} \delta c^{s} \cdot\left[\boldsymbol{D}_{\mathrm{cc}} \cdot \boldsymbol{\nabla}\left(\Delta c^{s}\right)+\boldsymbol{Y}_{\mathrm{cc}} \Delta c^{s}\right]\right\rangle_{\square}=\left\langle\boldsymbol{\nabla} \delta c^{s} \cdot \boldsymbol{J}_{\mathrm{c}}^{(k)}\right\rangle_{\square},
\end{aligned}
$$

for all possible $\delta \phi^{s}, \delta c^{s}$ that are sufficiently regular and which vanish on $\Gamma_{\square}$.

\section{3 $\quad \mathrm{FE}^{2}$ - Macroscale tangent operators}

In the general situation it is necessary to solve the macroscale problem Eqs. (3) and (4) and the corresponding RVE-problem in each macroscale quadrature point (typically Gauss points) in a "nested" fashion. The nested problem is conveniently solved via Newton iterations on the macroscale. Moreover, the procedure is commonly known as $\mathrm{FE}^{2}$ since both macroscale and subscale fields will be FE-discretized in $\Omega$ and $\Omega_{\square}$, respectively. It is then necessary

to find the macroscale tangent relations which represent linearizations of the (implicit) functions $\overline{\boldsymbol{J}}_{\phi}\left\{\bar{\phi}, \bar{c} ; \overline{\boldsymbol{g}}_{\phi}, \overline{\boldsymbol{g}}_{\mathrm{c}}\right\}$ and $\overline{\boldsymbol{J}}_{\mathrm{c}}\left\{\bar{\phi}, \bar{c} ; \overline{\boldsymbol{g}}_{\phi}, \overline{\boldsymbol{g}}_{\mathrm{c}}\right\}$ :

$$
\begin{aligned}
\mathrm{d} \overline{\boldsymbol{J}}_{\phi} & =-\overline{\boldsymbol{D}}_{\phi \phi} \cdot \mathrm{d} \overline{\boldsymbol{g}}_{\phi}-\overline{\boldsymbol{Y}}_{\phi \phi} \mathrm{d} \bar{\phi}-\overline{\boldsymbol{D}}_{\phi \mathrm{c}} \cdot \mathrm{d} \overline{\boldsymbol{g}}_{\mathrm{c}}-\overline{\boldsymbol{Y}}_{\phi \mathrm{c}} \mathrm{d} \bar{c}, \\
\mathrm{~d} \overline{\boldsymbol{J}}_{\mathrm{c}} & =-\overline{\boldsymbol{D}}_{\mathrm{c} \phi} \cdot \mathrm{d} \overline{\boldsymbol{g}}_{\phi}-\overline{\boldsymbol{Y}}_{\mathrm{c} \phi} \mathrm{d} \bar{\phi}-\overline{\boldsymbol{D}}_{\mathrm{cc}} \cdot \mathrm{d} \overline{\boldsymbol{g}}_{\mathrm{c}}-\overline{\boldsymbol{Y}}_{\mathrm{cc}} \mathrm{d} \bar{c}
\end{aligned}
$$

which requires the solution of the appropriate "sensitivity problem" (or tangent problem) associated with each RVE in each macroscale Newton iteration step. 
However, in this paper we shall not opt for the solution of the full-fledged nonlinear $\mathrm{FE}^{2}$ computations but rather make simplifications in order to derive upscaled macroscale constitutive relations. Such relations are obtained upon assuming linearization for vanishing macroscale spatial gradients, i.e. at the state $\overline{\boldsymbol{g}}_{\phi}=\overline{\boldsymbol{g}}_{\mathrm{c}}=\mathbf{0}$. The linearized expressions are then taken as valid for "small" changes of the macroscale spatial gradients.

\section{Linearized macroscopic problem}

\subsection{Preliminaries}

We shall henceforth consider the special situation that the state is defined by $\overline{\boldsymbol{g}}_{\phi}=\overline{\boldsymbol{g}}_{\mathrm{c}}=\mathbf{0}$. In such a case the RVE-problem with Dirichlet boundary conditions, although simplified, is still defined by the system in Eqs. (23) and (24); however, since we have assumed that $\overline{\boldsymbol{g}}_{\phi}=\overline{\boldsymbol{g}}_{\mathrm{c}}=\mathbf{0}$ we obtain $\phi^{M}=\bar{\phi}, c^{M}=\bar{c}$. Hence, $\phi=\bar{\phi}+\phi^{s}, c=\bar{c}+c^{s}$, and the constitutive relations Eqs. (5) and (6) become

$$
\begin{aligned}
& \boldsymbol{J}_{\phi}(\phi, c ; \boldsymbol{\nabla} \phi, \boldsymbol{\nabla} c)=-D_{\phi}(\phi) \boldsymbol{\nabla} \phi^{s}-\varepsilon_{\mathrm{c}} D_{\mathrm{c}}(\phi, c) \boldsymbol{\nabla} c^{s}, \\
& \boldsymbol{J}_{\mathrm{c}}(\phi, c ; \boldsymbol{\nabla} \phi, \boldsymbol{\nabla} c)=-\varepsilon_{\phi} D_{\phi}(\phi) \boldsymbol{\nabla} \phi^{s}-D_{\mathrm{c}}(\phi, c) \boldsymbol{\nabla} c^{s} .
\end{aligned}
$$

Upon inserting these relations into Eqs. (23) and (24), we obtain the RVEproblem

$$
\begin{gathered}
\left\langle D_{\phi} \boldsymbol{\nabla}\left(\delta \phi^{s}\right) \cdot \nabla \phi^{s}\right\rangle_{\square}+\varepsilon_{\mathrm{c}}\left\langle D_{\mathrm{c}} \boldsymbol{\nabla}\left(\delta \phi^{s}\right) \cdot \boldsymbol{\nabla} c^{s}\right\rangle_{\square}=0, \\
\varepsilon_{\phi}\left\langle D_{\phi} \boldsymbol{\nabla}\left(\delta c^{s}\right) \cdot \boldsymbol{\nabla} \phi^{s}\right\rangle_{\square}+\left\langle D_{\mathrm{c}} \boldsymbol{\nabla}\left(\delta c^{s}\right) \cdot \boldsymbol{\nabla} c^{s}\right\rangle_{\square}=0 .
\end{gathered}
$$

Upon setting $\delta \phi^{s}=\delta c^{s}=\phi^{s}$ in Eqs. (35) and (36) and eliminating the "mixed terms", we obtain

$$
\left[1-\varepsilon_{\phi} \varepsilon_{\mathrm{c}}\right]\left\langle D_{\phi}\left|\nabla \phi^{s}\right|^{2}\right\rangle_{\square}=0
$$

Now, since $\varepsilon_{\phi} \varepsilon_{\mathrm{c}} \neq 1$, due to the "compound" positiveness (as discussed in Section 2.3.2), we obtain the result $\left\langle D_{\phi}\left|\boldsymbol{\nabla} \phi^{s}\right|^{2}\right\rangle_{\square}=0$. Since $\phi^{s}$ has homogenous boundary conditions on $\Gamma_{\square}$, we conclude that the only possible 
solution is the homogeneous one, i.e. $\phi^{s}=0$. Similarly, by choosing $\delta \phi^{s}=$ $\delta c^{s}=c^{s}$ in Eqs. (35) and (36), we obtain the result $c^{s}=0$. Hence, $\phi=\bar{\phi}$ and $c=\bar{c}$ are constant fields within the RVE. In particular, we note that $\boldsymbol{\nabla} \phi=\boldsymbol{\nabla} c=\mathbf{0}$; hence, $\boldsymbol{Y}_{\phi \phi}=\boldsymbol{Y}_{\phi \mathrm{c}}=\boldsymbol{Y}_{\mathrm{c} \phi}=\boldsymbol{Y}_{\mathrm{cc}}=\mathbf{0}$.

As a result, in this very special case the linearized constitutive relations in Eqs. (25) and (26) reduce to

$$
\begin{aligned}
\mathrm{d} \boldsymbol{J}_{\phi} & =-D_{\phi} \boldsymbol{\nabla}(\mathrm{d} \phi)-\varepsilon_{\mathrm{c}} D_{\mathrm{c}} \boldsymbol{\nabla}(\mathrm{d} c), \\
\mathrm{d} \boldsymbol{J}_{\mathrm{c}} & =-\varepsilon_{\phi} D_{\phi} \boldsymbol{\nabla}(\mathrm{d} \phi)-D_{\mathrm{c}} \boldsymbol{\nabla}(\mathrm{d} c) .
\end{aligned}
$$

The sensitivity problem corresponding to variations of the macroscale fields $\bar{\phi}, \bar{c}$ and $\overline{\boldsymbol{g}}_{\phi}, \overline{\boldsymbol{g}}_{\mathrm{c}}$ can be expressed as

$$
\begin{aligned}
\left\langle\boldsymbol{\nabla}\left[\delta \phi^{s}\right] \cdot \mathrm{d} \boldsymbol{J}_{\phi}\right\rangle_{\square} & =0, \\
\left\langle\boldsymbol{\nabla}\left[\delta c^{s}\right] \cdot \mathrm{d} \boldsymbol{J}_{\mathrm{c}}\right\rangle_{\square} & =0,
\end{aligned}
$$

for all possible $\delta \phi^{s}, \delta c^{s}$ that are sufficiently regular and which vanish on $\Gamma_{\square}$. Upon inserting Eqs. (38) and (39) into Eqs. (40) and (41), we obtain the tangent relation

$$
\begin{aligned}
& \left\langle D_{\phi} \boldsymbol{\nabla}\left(\delta \phi^{s}\right) \cdot \boldsymbol{\nabla}(\mathrm{d} \phi)\right\rangle_{\square}+\varepsilon_{\mathrm{c}}\left\langle D_{\mathrm{c}} \boldsymbol{\nabla}\left(\delta \phi^{s}\right) \cdot \boldsymbol{\nabla}(\mathrm{d} c)\right\rangle_{\square}=0 \\
& \varepsilon_{\phi}\left\langle D_{\phi} \boldsymbol{\nabla}\left(\delta c^{s}\right) \cdot \boldsymbol{\nabla}(\mathrm{d} \phi)\right\rangle_{\square}+\left\langle D_{\mathrm{c}} \boldsymbol{\nabla}\left(\delta c^{s}\right) \cdot \boldsymbol{\nabla}(\mathrm{d} c)\right\rangle_{\square}=0 .
\end{aligned}
$$

\subsection{Sensitivity fields for SVE-problem}

To determine the relations in Eqs. (31) and (32) explicitly, we shall be interested in computing unit fluctuation fields, i.e. sensitivity fields, corresponding to unit values of $\mathrm{d} \bar{\phi}, \mathrm{d} \overline{\boldsymbol{g}}_{\phi}$ and $\mathrm{d} \bar{c}, \mathrm{~d} \overline{\boldsymbol{g}}_{\mathrm{c}}$. Hence, we need to compute the differentials $\mathrm{d} \phi=\mathrm{d} \phi^{M}+\mathrm{d} \phi^{s}$ and $\mathrm{d} c=\mathrm{d} c^{M}+\mathrm{d} c^{s}$ in terms of $\mathrm{d} \bar{\phi}, \mathrm{d} \overline{\boldsymbol{g}}_{\phi}$ and $\mathrm{d} \bar{c}, \mathrm{~d} \overline{\boldsymbol{g}}_{\mathrm{c}}$. Firstly, we conclude that

$$
\begin{aligned}
\mathrm{d} \phi^{M}(\boldsymbol{x}) & =\mathrm{d} \bar{\phi}+\mathrm{d} \overline{\boldsymbol{g}}_{\phi} \cdot[\boldsymbol{x}-\overline{\boldsymbol{x}}]=\sum_{i=1}^{N D I M} \hat{\phi}^{M(i)}(\boldsymbol{x}) \mathrm{d} \bar{g}_{H, i}, \\
\mathrm{~d} c^{M}(\boldsymbol{x}) & =\mathrm{d} \bar{c}+\mathrm{d} \overline{\boldsymbol{g}}_{\mathrm{c}} \cdot[\boldsymbol{x}-\overline{\boldsymbol{x}}]=\sum_{i=1}^{N D I M} \hat{c}^{M(i)}(\boldsymbol{x}) \mathrm{d} \bar{g}_{C, i},
\end{aligned}
$$


where $N D I M$ is the number of spatial dimensions. The unit fields, $\hat{\phi}^{M(i)}(\boldsymbol{x})$ and $\hat{c}^{M(i)}(\boldsymbol{x})$, are given as

$\hat{\phi}^{M(i)}(\boldsymbol{x})=\hat{c}^{M(i)}(\boldsymbol{x})=\boldsymbol{e}_{i} \cdot[\boldsymbol{x}-\overline{\boldsymbol{x}}]=x_{i}-\bar{x}_{i} \quad \Rightarrow \quad \boldsymbol{\nabla} \hat{\phi}^{M(i)}(\boldsymbol{x})=\boldsymbol{\nabla} \hat{c}^{M(i)}(\boldsymbol{x})=\boldsymbol{e}_{i}$.

Next, we introduce the following ansatz for $\mathrm{d} \phi^{s}$ and $\mathrm{d} c^{s}$ :

$$
\begin{aligned}
\mathrm{d} \phi^{s}(\boldsymbol{x}) & =\hat{\phi}_{\phi}^{s}(\boldsymbol{x}) \mathrm{d} \bar{\phi}+\hat{\phi}_{\mathrm{c}}^{s}(\boldsymbol{x}) \mathrm{d} \bar{c}+\sum_{i=1}^{N D I M} \hat{\phi}_{\phi \phi}^{s(i)}(\boldsymbol{x}) \mathrm{d} \bar{g}_{\phi, i}+\sum_{i=1}^{N D I M} \hat{\phi}_{\phi \mathrm{c}}^{s(i)}(\boldsymbol{x}) \mathrm{d} \bar{g}_{\mathrm{c}, i} \\
\mathrm{~d} c^{s}(\boldsymbol{x}) & =\hat{c}_{\mathrm{c}}^{s}(\boldsymbol{x}) \mathrm{d} \bar{c}+\hat{c}_{\phi}^{s}(\boldsymbol{x}) \mathrm{d} \bar{\phi}+\sum_{i=1}^{N D I M} \hat{c}_{\mathrm{cc}}^{s(i)}(\boldsymbol{x}) \mathrm{d} \bar{g}_{\mathrm{c}, i}+\sum_{i=1}^{N D I M} \hat{c}_{\mathrm{c} \phi}^{s(i)}(\boldsymbol{x}) \mathrm{d} \bar{g}_{\phi, i}
\end{aligned}
$$

We thus obtain the expressions for the total differentials, $\mathrm{d} \phi$ and $\mathrm{d} c$, in terms of the sensitivities as

$$
\begin{aligned}
\mathrm{d} \phi(\boldsymbol{x}) & =\left[1+\hat{\phi}_{\phi}^{s}(\boldsymbol{x})\right] \mathrm{d} \bar{\phi}+\hat{\phi}_{\mathrm{c}}^{s}(\boldsymbol{x}) \mathrm{d} \bar{c}+\sum_{i=1}^{N D I M}\left(\left[\hat{\phi}^{M(i)}(\boldsymbol{x})+\hat{\phi}_{\phi \phi}^{s(i)}(\boldsymbol{x})\right] \mathrm{d} \bar{g}_{\phi, i}+\hat{\phi}_{\phi \mathrm{c}}^{s(i)}(\boldsymbol{x}) \mathrm{d} \bar{g}_{\mathrm{c}, i}\right) \\
\mathrm{d} c(\boldsymbol{x}) & =\left[1+\hat{c}_{\mathrm{c}}^{s}(\boldsymbol{x})\right] \mathrm{d} \bar{c}+\hat{c}_{\phi}^{s}(\boldsymbol{x}) \mathrm{d} \bar{\phi}+\sum_{i=1}^{N D I M}\left(\left[\hat{c}^{M(i)}(\boldsymbol{x})+\hat{c}_{\mathrm{cc}}^{s(i)}(\boldsymbol{x})\right] \mathrm{d} \bar{g}_{\mathrm{c}, i}+\hat{c}_{\mathrm{c} \phi}^{s(i)}(\boldsymbol{x}) \mathrm{d} \bar{g}_{\phi, i}\right) .
\end{aligned}
$$

First, we show that the sensitivities $\hat{\phi}_{\phi}^{s}, \hat{\phi}_{\mathrm{c}}^{s}, \hat{c}_{\phi}^{s}$ and $\hat{c}_{\mathrm{c}}^{s}$ all vanish. To this end, consider first the case $\mathrm{d} \bar{\phi} \neq \mathbf{0}$ while all other macroscale differentials are zero. Upon inserting the expressions in Eqs. (49) and (50) into Eqs. (42) and (43), we identify the coupled system

$$
\begin{aligned}
& \left\langle D_{\phi} \nabla\left(\delta \phi^{s}\right) \cdot \nabla \hat{\phi}_{\phi}^{s}\right\rangle_{\square}+\varepsilon_{\mathrm{c}}\left\langle D_{\mathrm{c}} \boldsymbol{\nabla}\left(\delta \phi^{s}\right) \cdot \nabla \hat{c}_{\phi}^{s}\right\rangle_{\square}=0, \\
& \varepsilon_{\phi}\left\langle D_{\phi} \boldsymbol{\nabla}\left(\delta c^{s}\right) \cdot \nabla \hat{\phi}_{\phi}^{s}\right\rangle_{\square}+\left\langle D_{\mathrm{c}} \boldsymbol{\nabla}\left(\delta c^{s}\right) \cdot \nabla \hat{c}_{\phi}^{s}\right\rangle_{\square}=0 .
\end{aligned}
$$


Setting $\delta \phi^{s}=\delta c^{s}=\hat{c}_{\phi}^{s}$ in Eqs. (51) and (52), we obtain

$$
\left[1-\varepsilon_{\phi} \varepsilon_{\mathrm{c}}\right]\left\langle D_{\mathrm{c}}\left|\nabla \hat{c}_{\phi}^{s}\right|^{2}\right\rangle_{\square}=0 .
$$

Thus, since $\varepsilon_{\phi} \varepsilon_{\mathrm{c}} \neq 1$, due to the "compound" positive definiteness (as discussed in Section 2.3.2), we obtain the result $\left\langle D_{\mathrm{c}}\left|\boldsymbol{\nabla} \hat{c}_{\phi}^{s}\right|^{2}\right\rangle_{\square}=0$ by which we, finally, conclude that $\hat{c}_{\phi}^{s}=0$. It then follows directly that $\hat{\phi}_{\phi}^{s}=0$. In a similar fashion, by considering the situation $\mathrm{d} \bar{c} \neq 0$ while all other macroscale differentials are zero, we obtain $\hat{\phi}_{\mathrm{c}}^{s}=\hat{c}_{\mathrm{c}}^{s}=0$.

Next, we shall show that the two "coupling sensitivities", $\hat{\phi}_{\phi c}^{s(i)}(\boldsymbol{x})$ and $\hat{c}_{\mathrm{c} \phi}^{s(i)}(\boldsymbol{x})$, both vanish; hence, the system in Eqs. (42) and (43) will be completely decoupled. Consider then first the case $\mathrm{d} \overline{\boldsymbol{g}}_{\phi} \neq \mathbf{0}, \mathrm{d} \overline{\boldsymbol{g}}_{\mathrm{c}}=\mathbf{0}$. Upon inserting the expressions in Eqs. (49) and (50) into Eqs. (42) and (43), we identify the coupled system

$$
\begin{aligned}
& \left\langle D_{\phi} \boldsymbol{\nabla}\left(\delta \phi^{s}\right) \cdot \nabla \hat{\phi}_{\phi \phi}^{s(i)}\right\rangle_{\square}+\varepsilon_{\mathrm{c}}\left\langle D_{\mathrm{c}} \boldsymbol{\nabla}\left(\delta \phi^{s}\right) \cdot \nabla \hat{c}_{\mathrm{c} \phi}^{s(i)}\right\rangle_{\square}=-\left\langle D_{\phi} \boldsymbol{\nabla}\left(\delta \phi^{s}\right) \cdot \boldsymbol{e}_{i}\right\rangle_{\square} \\
& \varepsilon_{\phi}\left\langle D_{\phi} \boldsymbol{\nabla}\left(\delta c^{s}\right) \cdot \boldsymbol{\nabla} \hat{\phi}_{\phi \phi}^{s(i)}\right\rangle_{\square}+\left\langle D_{\mathrm{c}} \boldsymbol{\nabla}\left(\delta c^{s}\right) \cdot \nabla \hat{c}_{\mathrm{c} \phi}^{s(i)}\right\rangle_{\square}=-\varepsilon_{\phi}\left\langle D_{\phi} \boldsymbol{\nabla}\left(\delta c^{s}\right) \cdot \boldsymbol{e}_{i}\right\rangle_{\square} .
\end{aligned}
$$

Now, setting $\delta \phi^{s}=\delta c^{s}=\hat{c}_{\mathrm{c} \phi}^{s(i)}$ in Eqs. (54) and (55), we may eliminate the "mixed" terms to obtain

$$
\left[1-\varepsilon_{\phi} \varepsilon_{\mathrm{c}}\right]\left\langle D_{\mathrm{c}}\left|\nabla \hat{c}_{\mathrm{c} \phi}^{s(i)}\right|^{2}\right\rangle_{\square}=0
$$

Thus, since $\varepsilon_{\phi} \varepsilon_{\mathrm{c}} \neq 1$, we obtain the result $\left\langle D_{\mathrm{c}}\left|\nabla \hat{c}_{\mathrm{c} \phi}^{s(i)}\right|^{2}\right\rangle_{\square}=0$ by which we, finally, conclude that $\hat{c}_{\mathrm{c} \phi}^{s(i)}=0$.

In a similar fashion, by considering, the case $\mathrm{d} \overline{\boldsymbol{g}}_{\phi}=\mathbf{0}, \mathrm{d} \overline{\boldsymbol{g}}_{\mathrm{c}} \neq \mathbf{0}$, we obtain that $\hat{\phi}_{\phi \mathrm{c}}^{s(i)}=0$.

In summary, we have 


$$
\begin{aligned}
\mathrm{d} \phi(\boldsymbol{x}) & =\sum_{i=1}^{N D I M}\left[\hat{\phi}^{M(i)}(\boldsymbol{x})+\hat{\phi}^{s(i)}(\boldsymbol{x})\right] \mathrm{d} \bar{g}_{\phi, i}, \\
\mathrm{~d} c(\boldsymbol{x}) & =\sum_{i=1}^{N D I M}\left[\hat{c}^{M(i)}(\boldsymbol{x})+\hat{c}^{s(i)}(\boldsymbol{x})\right] \mathrm{d} \bar{g}_{\mathrm{c}, i},
\end{aligned}
$$

where the simplified notation $\hat{\phi}^{s(i)} \stackrel{\text { def }}{=} \hat{\phi}_{\phi \phi}^{s(i)}$ and $\hat{c}^{s(i)} \stackrel{\text { def }}{=} \hat{c}_{\mathrm{cc}}^{s(i)}$ was introduced. We thus conclude that the sensitivity fields due to changes in $\overline{\boldsymbol{g}}_{\phi}$ and $\overline{\boldsymbol{g}}_{\mathrm{c}}$, respectively, are uncoupled.

Remark 4 The decoupling is due to the specific assumptions made here and does not hold for the general nonlinear problem defined by Eqs. (23) and (24).

The remaining (uncoupled) equations become

$$
\begin{aligned}
\left\langle D_{\phi} \boldsymbol{\nabla}\left(\delta \phi^{s}\right) \cdot \boldsymbol{\nabla} \hat{\phi}^{s(i)}\right\rangle_{\square} & =-\left\langle D_{\phi} \boldsymbol{\nabla}\left(\delta \phi^{s}\right) \cdot \boldsymbol{e}_{i}\right\rangle_{\square} \\
\left\langle D_{\mathrm{c}} \boldsymbol{\nabla}\left(\delta c^{s}\right) \cdot \boldsymbol{\nabla} \hat{c}^{s(i)}\right\rangle_{\square} & =-\left\langle D_{\mathrm{c}} \boldsymbol{\nabla}\left(\delta c^{s}\right) \cdot \boldsymbol{e}_{i}\right\rangle_{\square},
\end{aligned}
$$

from which $\hat{\phi}^{s(i)}$ and $\hat{c}^{s(i)}$ can be computed.

The expressions in Eqs. (59) and (60) are valid in the general situation where the subscale properties are allowed to be inhomogeneous within the constituents in a completely general fashion. However, an interesting simplification arises in the following important special case: The material parameters characterizing the diffusion coefficients $D_{\phi}$ and $D_{\mathrm{c}}$, which are "intrinsic" to the cement paste, are homogeneous within the subdomain $\Omega_{\square, \mathrm{cp}}{ }^{5}$, and it is assumed that the diffusivities are zero in the subdomain $\Omega_{\square, \mathrm{b}}$ occupied by the ballast particles. Since $\phi=\bar{\phi}$ and $c=\bar{c}$ are constant fields within the SVE, we conclude that $\phi$ and $c$ are indeed homogeneous within $\Omega_{\square, \mathrm{cp}}$; hence, we may set

$$
D_{\phi}(\boldsymbol{x})=D_{\phi, \mathrm{cp}} f(\boldsymbol{x}) ; \quad D_{\mathrm{c}}(\boldsymbol{x})=D_{\mathrm{c}, \mathrm{cp}} f(\boldsymbol{x}),
$$

\footnotetext{
${ }^{5}$ sub-indices "cp" for cement paste and "b" for ballast.
} 
with the common function

$$
f(\boldsymbol{x})= \begin{cases}1, & \forall \boldsymbol{x} \in \Omega_{\square, \mathrm{cp}} \\ 0, & \forall \boldsymbol{x} \in \Omega_{\square, \mathrm{b}}\end{cases}
$$

in Eqs. (59) and (60) to obtain the two identical equations

$$
\begin{gathered}
\left\langle f \nabla\left(\delta \phi^{s}\right) \cdot \nabla \hat{\phi}^{s(i)}\right\rangle_{\square}=-\left\langle f \nabla\left(\delta \phi^{s}\right) \cdot \boldsymbol{e}_{i}\right\rangle_{\square}, \\
\left\langle f \nabla\left(\delta c^{s}\right) \cdot \nabla \hat{c}^{s(i)}\right\rangle_{\square}=-\left\langle f \nabla\left(\delta c^{s}\right) \cdot \boldsymbol{e}_{i}\right\rangle_{\square},
\end{gathered}
$$

for the unknown sensitivity fields $\hat{\phi}^{s(i)}$ and $\hat{c}^{s(i)}$; hence, we conclude that these fields are identical.

\subsection{Homogenized linearized diffusivity tensors}

We are, finally, in the position to evaluate the macroscale diffusivity tensors (defined via linearization) occurring in Eqs. (31) and (32) by first noting the identities

$$
\begin{aligned}
\mathrm{d} \bar{J}_{\phi, i} & =\left\langle\mathrm{d} \boldsymbol{J}_{\phi} \cdot \boldsymbol{e}_{i}\right\rangle_{\square}, \\
\mathrm{d} \bar{J}_{\mathrm{c}, i} & =\left\langle\mathrm{d} \boldsymbol{J}_{\mathrm{c}} \cdot \boldsymbol{e}_{i}\right\rangle_{\square},
\end{aligned}
$$

and combining with Eqs. (38) and (39) and Eqs. (57) and (58) to obtain (i) $\overline{\boldsymbol{Y}}_{\phi \phi}=\overline{\boldsymbol{Y}}_{\phi \mathrm{c}}=\overline{\boldsymbol{Y}}_{\mathrm{c} \phi}=\overline{\boldsymbol{Y}}_{\mathrm{cc}}=\mathbf{0}$ and (ii) the non-zero tangent tensors in terms of their components

$$
\begin{aligned}
\left(\overline{\boldsymbol{D}}_{\phi \phi}\right)_{i j} & =\left\langle D_{\phi}\right\rangle_{\square} \delta_{i j}+\left\langle D_{\phi} \boldsymbol{\nabla}\left(\hat{\phi}^{s(j)}\right) \cdot \boldsymbol{e}_{i}\right\rangle_{\square}, \\
\left(\overline{\boldsymbol{D}}_{\phi \mathrm{c}}\right)_{i j} & =\varepsilon_{\mathrm{c}}\left\langle D_{\mathrm{c}}\right\rangle_{\square} \delta_{i j}+\varepsilon_{\mathrm{c}}\left\langle D_{\mathrm{c}} \boldsymbol{\nabla}\left(\hat{c}^{s(j)}\right) \cdot \boldsymbol{e}_{i}\right\rangle_{\square}, \\
\left(\overline{\boldsymbol{D}}_{\mathrm{c} \phi}\right)_{i j} & =\varepsilon_{\phi}\left\langle D_{\phi}\right\rangle_{\square} \delta_{i j}+\varepsilon_{\phi}\left\langle D_{\phi} \boldsymbol{\nabla}\left(\hat{\phi}^{s(j)}\right) \cdot \boldsymbol{e}_{i}\right\rangle_{\square}, \\
\left(\overline{\boldsymbol{D}}_{\mathrm{cc}}\right)_{i j} & =\left\langle D_{\mathrm{c}}\right\rangle_{\square} \delta_{i j}+\left\langle D_{\mathrm{c}} \nabla\left(\hat{c}^{s(j)}\right) \cdot \boldsymbol{e}_{i}\right\rangle_{\square}, \\
& \overline{\boldsymbol{D}}_{\phi \mathrm{c}}=\varepsilon_{\mathrm{c}} \overline{\boldsymbol{D}}_{\mathrm{cc}}, \quad \overline{\boldsymbol{D}}_{\mathrm{c} \phi}=\varepsilon_{\phi} \overline{\boldsymbol{D}}_{\phi \phi} .
\end{aligned}
$$


For small spatial gradients, we thus propose the approximate constitutive relations for $\overline{\boldsymbol{J}}_{\phi}$ and $\overline{\boldsymbol{J}}_{\mathrm{c}}$ as follows:

$$
\begin{aligned}
& \overline{\boldsymbol{J}}_{\phi} \approx-\overline{\boldsymbol{D}}_{\phi} \cdot \overline{\boldsymbol{\nabla}} \bar{\phi}-\varepsilon_{\mathrm{c}} \overline{\boldsymbol{D}}_{\mathrm{c}} \cdot \overline{\boldsymbol{\nabla}} \bar{c} \\
& \overline{\boldsymbol{J}}_{\mathrm{c}} \approx-\varepsilon_{\phi} \overline{\boldsymbol{D}}_{\phi} \cdot \overline{\boldsymbol{\nabla}} \bar{\phi}-\overline{\boldsymbol{D}}_{\mathrm{c}} \cdot \overline{\boldsymbol{\nabla}} \bar{c}
\end{aligned}
$$

where we introduced the simplified notation $\overline{\boldsymbol{D}}_{\phi} \stackrel{\text { def }}{=} \overline{\boldsymbol{D}}_{\phi \phi}$ and $\overline{\boldsymbol{D}}_{\mathrm{c}} \stackrel{\text { def }}{=} \overline{\boldsymbol{D}}_{\mathrm{cc}}$.

We may, further, get a more explicit expression of $\overline{\boldsymbol{D}}_{\phi}$ and $\overline{\boldsymbol{D}}_{\mathrm{c}}$ when the parameters $D_{\phi}$ and $D_{\mathrm{c}}$ are homogeneous within the subdomain $\Omega_{\square, \mathrm{cp}}$ (as discussed above). It then follows that

$$
\left(\overline{\boldsymbol{D}}_{\phi}\right)_{i j}=\left[1-n_{\mathrm{b}}\right] D_{\phi, \mathrm{cp}}\left[\delta_{i j}+\left\langle\boldsymbol{\nabla} \hat{\phi}^{s(j)} \cdot \boldsymbol{e}_{i}\right\rangle_{\square, \mathrm{cp}}\right],
$$

where we introduced the "phase average"

$$
\langle\bullet\rangle_{\square, \mathrm{cp}}=\frac{1}{\left|\Omega_{\square, \mathrm{cp}}\right|} \int_{\Omega_{\square, \mathrm{cp}}} \bullet \mathrm{d} \Omega_{\square} .
$$

In the case of macroscopic isotropy, which should be retrieved in the present setup for sufficiently large $L_{\square}$, then it is possible to represent the "fluctuation" part as

$$
\left\langle\boldsymbol{\nabla} \hat{\phi}^{s(j)} \cdot \boldsymbol{e}_{i}\right\rangle_{\square}=\left\langle\boldsymbol{\nabla} \hat{c}^{s(j)} \cdot \boldsymbol{e}_{i}\right\rangle_{\square}=\bar{A}^{s} \delta_{i j}
$$

where

$$
\bar{A}^{s}=\left(\left\langle\boldsymbol{\nabla} \hat{\phi}^{s(1)}\right\rangle_{\square}\right)_{1}=\left(\left\langle\boldsymbol{\nabla} \hat{\phi}^{s(2)}\right\rangle_{\square}\right)_{2}=\left(\left\langle\nabla \hat{c}^{s(1)}\right\rangle_{\square}\right)_{1}=\left(\left\langle\nabla \hat{c}^{s(2)}\right\rangle_{\square}\right)_{2} .
$$

Hence, we may write

$$
\overline{\boldsymbol{D}}_{\phi}=\bar{D}_{\phi} \boldsymbol{I}, \quad \text { with } \quad \bar{D}_{\phi}=\left[1-n_{\mathrm{b}}\right]\left[1+\bar{A}^{s}\right] D_{\phi, \mathrm{cp}} .
$$

Obviously, a similar expression can be obtained for $\overline{\boldsymbol{D}}_{\mathrm{c}}$ : 


$$
\overline{\boldsymbol{D}}_{\mathrm{c}}=\bar{D}_{\mathrm{c}} \boldsymbol{I}, \quad \text { with } \quad \bar{D}_{\mathrm{c}}=\left[1-n_{\mathrm{b}}\right]\left[1+\bar{A}^{s}\right] D_{\mathrm{c}, \mathrm{cp}} .
$$

An estimate of the Hashin-Shtrikman type, which was adopted in [3, 21], for the effective diffusion coefficients of concrete, $\bar{D}_{\phi}$ and $\bar{D}_{\mathrm{c}}$, can be obtained by multiplying $D_{\phi, \text { cp }}$ and $D_{\text {c,cp }}$ by a common factor $f\left(n_{\mathrm{b}}\right)$ depending only on the ballast volume fraction $n_{\mathrm{b}}$, i.e.

$$
\bar{D}_{\phi}=f\left(n_{\mathrm{b}}\right) D_{\phi, \mathrm{cp}}, \quad \bar{D}_{\mathrm{c}}=f\left(n_{\mathrm{b}}\right) D_{\mathrm{c}, \mathrm{cp}},
$$

where the explicit expression for $f\left(n_{\mathrm{b}}\right)$ is

$$
f\left(n_{\mathrm{b}}\right)=\frac{2-2 n_{\mathrm{b}}}{2+n_{\mathrm{b}}} \quad \forall n_{\mathrm{b}} \in[0,1] .
$$

Clearly, the Voigt bounds are given by $\bar{A}^{s}=0$ in Eq. (78) and Eq. (79). The actual results are later, in Section 5.2, compared with the HS-estimate in Eq. (78) as well as the Voigt bound.

\section{Computational results}

All computational implementation carried out for this paper, including FEmeshing, was done in MATLAB ${ }^{\circledR}$ R2008B.

\subsection{RVE size}

Computations of homogenized flux quantities were conducted first in order to estimate the appropriate RVE size. By imposing Dirichlet boundary conditions based on prescribed values of $\bar{\nabla} \bar{\phi}$ and $\bar{\nabla} \bar{c}$, respectively, the stationary problems were solved for SVEs of varying side length, $L_{\square}$. In Figure 6 , snapshots of the fields $\phi(\boldsymbol{x})$ and $c(\boldsymbol{x})$ are shown for gradients in directions 1 and 2 , respectively. 


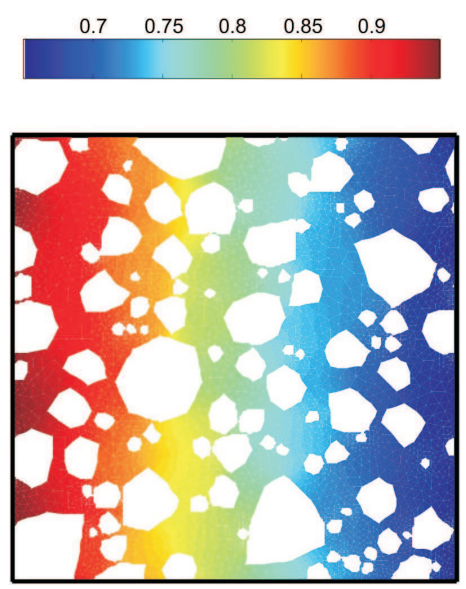

(a) Relative humidity for $[\bar{\nabla} \bar{\phi}]=$

$$
\left[\begin{array}{ll}
-1 & 0
\end{array}\right]^{\mathrm{T}}
$$
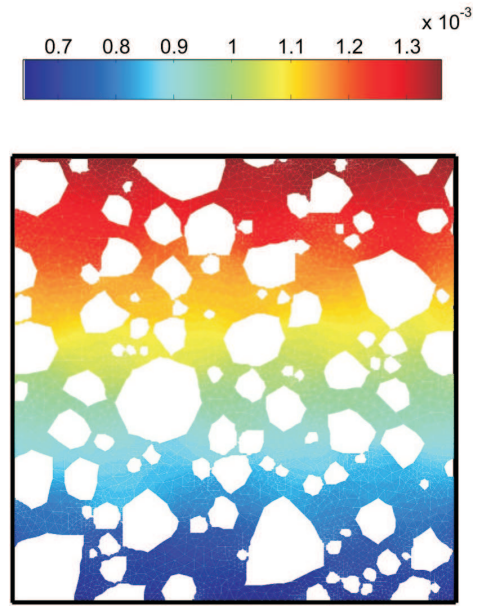

(b)

Chloride concentration for $[\bar{\nabla} \bar{c}]=$ $\left[\begin{array}{ll}0 & 1\end{array}\right]^{\mathrm{T}}$

Figure 6: Applied macroscale moisture and chloride ion gradients in direction 1 and 2 , respectively.

We are interested in constructing bounds on the numerical results in terms of confidence intervals. The confidence limits are given as

$$
\mu(\overline{\boldsymbol{J}}) \pm t_{\alpha / 2, N-1} \frac{\sigma(\overline{\boldsymbol{J}})}{\sqrt{N}},
$$

where $\mu$ denotes the mean value, $t_{\alpha / 2, N-1}$ is the upper critical value of the t-distribution with $N-1$ degrees of freedom, $\sigma$ is the standard deviation, and $N$ is the number of SVE realizations. In addition, $\alpha=1-\mathrm{CI}$ where CI is the confidence interval. 


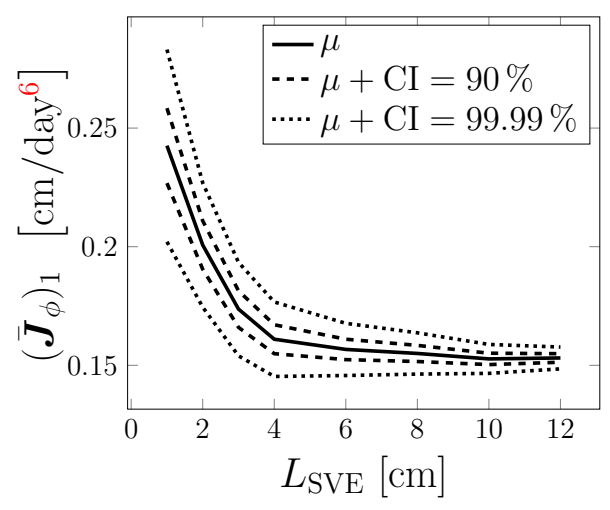

(a) Flux parallel to applied gradient.

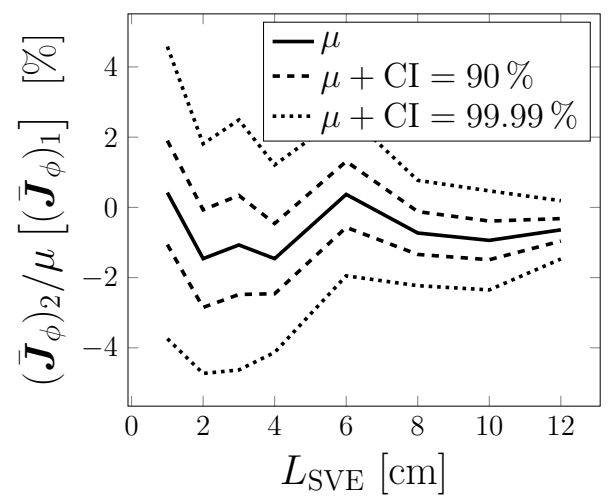

(b) Normalized flux perpendicular to applied gradient.

Figure 7: Homogenized flux as a function of SVE-size for $[\bar{\nabla} \bar{\phi}]=\left[\begin{array}{ll}-1 & 0\end{array}\right]^{T}$.

For the present study, we have chosen a sample size of 40 SVE realizations $(N=40)$, all with ballast content of $40 \%\left(n_{\mathrm{b}}=0.4\right)$. In Figure 7, components of the homogenized stationary flux $\overline{\boldsymbol{J}}_{\phi}$ are shown for varying SVE sizes. A unit value of the moisture gradient was applied in direction 1 , hence the component $\left(\overline{\boldsymbol{J}}_{\phi}\right)_{2}$ can be seen as a measure of the SVE's anisotropy. The SVE is anisotropic by construction; however, the homogenized response is expected to be isotropic for an infinitely large sample of SVE realizations, i.e. $\lim _{N \rightarrow \infty} \sum_{i=0}^{N}\left(\overline{\boldsymbol{J}}_{\phi}\right)_{2, i}=0$. Based on the results in Figure 7, it was decided to choose $L_{\mathrm{RVE}}=10[\mathrm{~cm}]$ as the side length of the RVE, used for further analyses. It should be noted that the size of the RVE, for which the converged value of $\left(\overline{\boldsymbol{J}}_{\phi}\right)_{1}$ is obtained, is highly dependent on the choice of the ballast sieve curve. A ballast sieve curve chosen with greater ballast radii would demand a larger $L_{\mathrm{RVE}}$.

\subsection{Effective material response - influence of ballast content}

Once an adequate RVE size was determined, stationary diffusion analyses were conducted for which the RVE size was kept constant; instead, the ballast

\footnotetext{
${ }^{6}$ Natively, the unit for flux is $\left[\mathrm{kg} / \mathrm{m}^{2} \mathrm{~s}\right]$ but here we have the unit of $\phi=[-]$; hence, $\boldsymbol{J}$ will have units of $[\mathrm{cm} /$ day $]$.
} 
content, $n_{\mathrm{b}}$, was allowed to vary. For these analyses the ballast sieve curve shown in Figure 2 was chosen.

By letting the ballast content, $n_{\mathrm{b}}$, vary within the RVE, and by utilizing the expressions of Eqs. (67) to (70), the dependence of $\overline{\boldsymbol{D}}_{\phi}$ and $\overline{\boldsymbol{D}}_{\mathrm{c}}$ on the ballast fraction could be determined. For these analyses, 20 RVEs were generated for each value of $n_{\mathrm{b}}$. In Figure $8 \mathrm{a},\left(\overline{\boldsymbol{D}}_{\phi}\right)_{11}$ and $\left(\overline{\boldsymbol{D}}_{\phi}\right)_{22}$ are given as functions of $n_{\mathrm{b}}$, whereas the corresponding relations for $\left(\overline{\boldsymbol{D}}_{\phi}\right)_{12}$ and $\left(\overline{\boldsymbol{D}}_{\phi}\right)_{21}$ are shown in Figure 8b. It is noteworthy that (i) $\mu\left[\left(\overline{\boldsymbol{D}}_{\phi}\right)_{11}\right] \approx \mu\left[\left(\overline{\boldsymbol{D}}_{\phi}\right)_{22}\right]$ confirms that the mean response of the RVEs is isotropic (the RVE is indeed sufficiently large), and that (ii) the result is in good agreement with the Hashin-Shtrikman (HS) estimate.

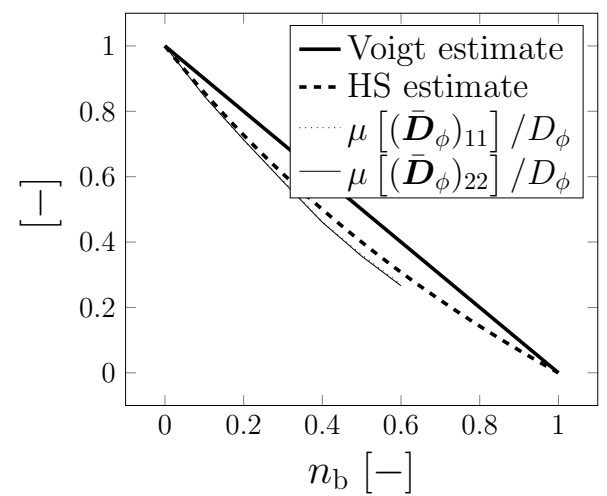

(a) Diagonal elements of $\overline{\boldsymbol{D}}_{\phi}$.

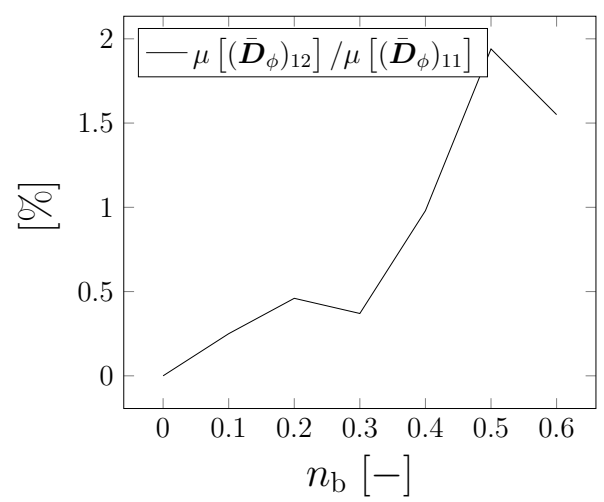

(b) Off-diagonal elements of $\overline{\boldsymbol{D}}_{\phi}$.

Figure 8: $\overline{\boldsymbol{D}}_{\phi}$ as a function of $n_{\mathrm{b}}$.

Remark 5 Note that the the Hashin-Shtrikman model scales the influence of ballast based on volume fraction, whereas this model is based on area fraction.

Remark 6 The "gap" between the Voigt estimate and the computed diffusivities in Figure 8 a corresponds to the the "fluctuation" term $\bar{A}^{s}$ in Eq. (78).

Remark 7 As to the difference between area-volume fraction models, we observe that effective Young's moduli for heterogenous cement paste were determined in [9] and it was concluded that there is a significant difference between $3 D$ and $2 D$ RVEs in terms of computational results. 


\subsection{The effect of ITZ}

So far, the ITZ has been excluded from the analyses. The same analyses used to determine $\overline{\boldsymbol{D}}_{\phi}$ and $\overline{\boldsymbol{D}}_{\text {c }}$ were repeated including the ITZ. In Figure 9 the influence of the ITZ on $\overline{\boldsymbol{D}}$ is shown for varying values of $D_{\text {ITZ }}$.

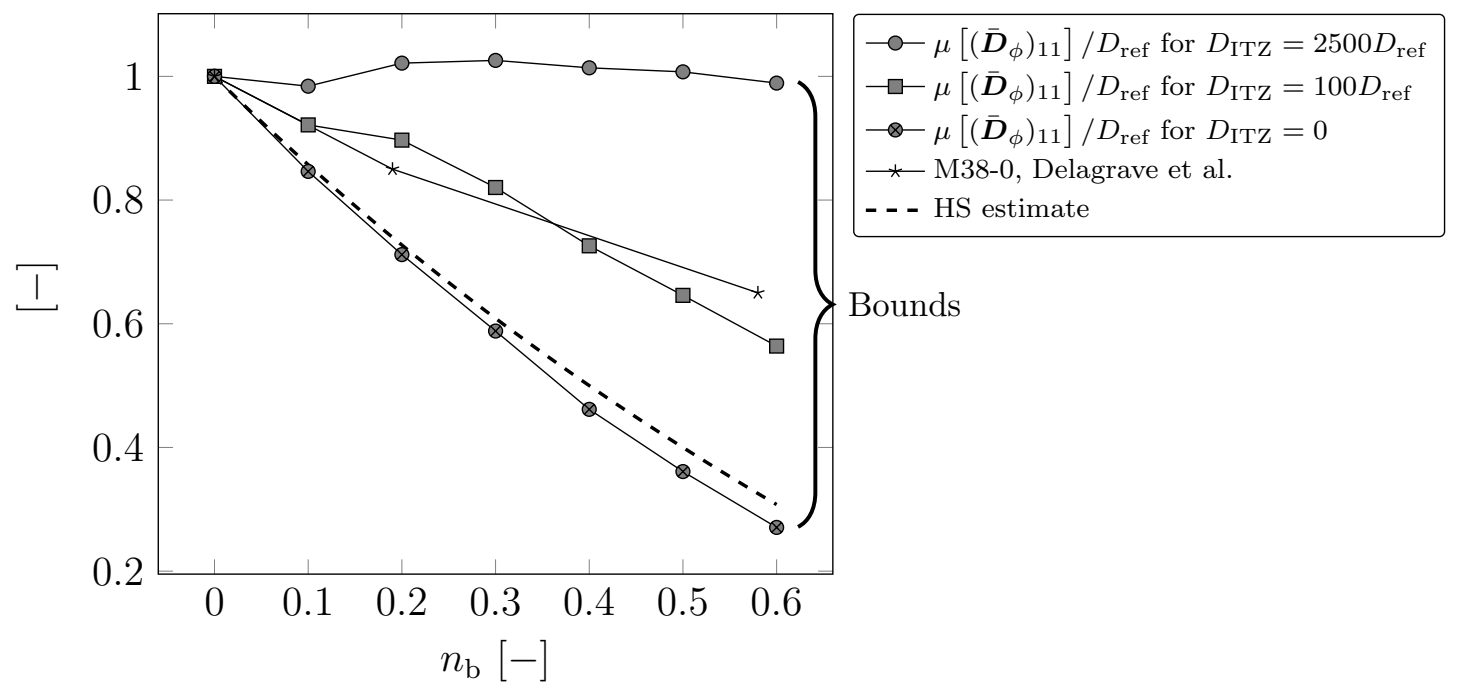

Figure 9: Effect of ITZ on the effective diffusivity. These relations hold for both $\phi$ and $c . D_{\text {ref }}$ denotes a reference diffusion coefficient for either $\phi$ or $c$ in the pure cement paste.

As Figure 9 shows, the value of $D_{\text {ITZ }}$ greatly influences the effective diffusivity properties of concrete; in the considered example of $D_{\mathrm{ITZ}}=2500 D_{\text {ref }}$, the ITZ totally eliminates the effect of the ballast, i.e. $\mu\left[\left(\overline{\boldsymbol{D}}_{\phi}\right)_{11}\right] \approx D_{\text {ref }}$. Here, $D_{\text {ref }}$ is a reference diffusivity of the pure cement paste. This shows the importance of having an accurately modeled mesoscale structure of concrete in order to execute proper diffusion simulations.

Experiments were conducted in [5] on mortars with variable sand volume contents to determine the influence of ITZ, and their results are included in Figure 9. It should be noted that their results are for sand-sized particles whereas the RVEs generated herein include gravel-sized particles. Furthermore, the effect of ITZ will be more predominant in sand-sized particles since the circumference to area ratio will be greater for sand particles than for ballast particles. Hence, the experimental data are included mainly to indicate that 
the ITZ apperars to have a noticeable affect on the effective diffusivity. From the computational results, the value of $D_{\text {ITZ }}$ appears to be in the vicinity of $100 D_{\text {ref }}$ in this linearized case that has been considered, see Figure 9.

Remark 8 The determined value of $100 D_{\text {ref }}$ is a direct consequence of the choice of $h=60 \mathrm{um}^{2}$. In fact, it is rather the product $D_{\text {ITZ }}$ that is determined by calibration with experimental data.

The upper and lower curves in Figure 9 denote the bounds of the influence of ITZ on the effective diffusivity. Thus, the real effect of $D_{\text {ITZ }}$ is expected to lie between these two curves. It should be noted that the upper bound is invariant to $D_{\text {ITZ }}>2500 D_{\text {ref }}$ (for the case $h=60 \mu \mathrm{m}^{2}$ ). However, the upper bound can take values $\gg 1$ if the ballast particles are in contact with each other, thus forming continuous 'pipes' of ITZ throughout the RVE.

\section{Conclusions and Outlook}

In this contribution, the effect of ballast on the diffusivity of concrete was evaluated by adopting the concepts of Representative Volume Element (RVE) and computational homogenization. Concrete was modeled as a three-phase composite consisting of the cement paste, ballast and the Interfacial Transition Zone (ITZ). The algorithm devised for generating RVEs provides the possibility to mimic the random shape and size of ballast, as well as to simulate ballast content of various sieve gradations and area fractions in an accurate way. The computational results show that the influence of ballast content corresponds well to the Hashi-Shtrikman estimate, cf. Figure 8a.

Furthermore, the model proposed here allows for modelling of the ITZ by including this highly porous phase as 1D interface elements in the FEdiscretized computational domain. The diffusivity coefficient, $D_{\text {ITZ }}$, was calibrated using experimental results of stationary fluxes of chloride ions. The bounds of the effect of ITZ were also determined for the effective diffusivity of concrete.

As an outlook for future work, the model will be included in an $\mathrm{FE}^{2}$ strategy for transient simulations in which the material response on the macroscale is obtained in a nested fashion by solving the RVE problem introduced in each macroscale Gauss-point. Consequently, the RVE problem will in this manner serve as a constitutive model on the macroscale level. 


\section{Acknowledgments}

This research was financially supported by The Swedish Research Council, which is gratefully acknowledged.

\section{References}

[1] A Ababneh, F Benboudjema, and Y Xi. Chloride penetration in nonsaturated concrete. J. Mater. Civ. Eng., 15(2):183-191, 2003.

[2] A Caballero, C López, and I Carol. 3D meso-structural analysis of concrete specimens under uniaxial tension. Comput. Methods Appl. Mech. Engrg., 195(52):7182-7195, 2006.

[3] R M Christensen. Mechanics of Composite Materials. Wiley Interscience, New York, 1979.

[4] A Cwirzen and V Penttala. Aggregate-cement paste transition zone properties affecting the salt-frost damage of high-performance concretes. Cement Concr. Res., 35(4):671-679, 2005.

[5] A Delagrave, J Bigas, J P Ollivier, J Marchand, and M Pigeon. Influence of the interfacial zone on the chloride diffusivity of mortars. Adv. Cem. Based Mater., 5(3-4):86-92, 1997.

[6] J Fish. Bridging the scales in nano engineering and science. Journal of Nanoparticle Research, 8(5):577-594, 2006.

[7] P Grassl. A lattice approach to model flow in cracked concrete. Cement and Concrete Comp., 31(7):454-460, 2009.

[8] P Grassl and M Jirásek. Damage-plastic model for concrete failure. Int. J. Solid. Struct., 43(22-23):7166-7196, 2006.

[9] M Hain and P Wriggers. Numerical homogenization of hardened cement paste. Computational Mechanics, 42(2):197-212, 2007.

[10] M Hain and P Wriggers. Computational homogenization of microstructural damage due to frost in hardened cement paste. Finite. Elem. Anal. Des., 44(5):233-244, 2008. 
[11] Z Hashin and S Shtrikman. A variational approach to the theory of the effective magnetic permeability of multiphase materials. Journal of Applied Physics, 33(10):3125, 1962.

[12] F Larsson, K Runesson, and F Su. Computational homogenization of uncoupled consolidation in micro-heterogeneous porous media. Int. J. Numer. Anal. Meth. Geomech., 34(14):1431-1458, 2009.

[13] K Liao, P Chang, Y Peng, and C Yang. A study on characteristics of interfacial transition zone in concrete. Cement Concr. Res., 34(6):977989, 2004.

[14] C Miehe and C G Bayreuther. On multiscale FE analyses of heterogeneous structures: From homogenization to multigrid solvers. Int. J. Numer. Meth. Eng., 71(10):1135-1180, 2007.

[15] TD Tran Ngoc, J Lewandowska, M Vauclin, and H Bertin. Two-scale modeling of solute dispersion in unsaturated double-porosity media: Homogenization and experimental validation. Int. J. Numer. Anal. Meth. Geomech., 35(14):1536-1559, October 2011.

[16] V Nguyen, B Nedjar, H Colina, and J Torrenti. A separation of scales homogenization analysis for the modelling of calcium leaching in concrete. Comput. Methods Appl. Mech. Engrg., 195(52):7196-7210, 2006.

[17] M A Peter. Coupled reaction-diffusion processes inducing an evolution of the microstructure: Analysis and homogenization. Nonlinear Anal. Theor. Meth. Appl., 70(2):806-821, 2009.

[18] J Rodriguez, L M Ortega, and J Casal. Load carrying capacity of concrete structures with corroded reinforcement. Constr Build Mater., 11(4):239-248, 1997.

[19] E Samson, J Marchand, and J J Beaudoin. Describing ion diffusion mechanisms in cement-based materials using the homogenization technique. Cement Concr. Res., 29(8):1341-1345, 1999.

[20] T Stankowski. Numerical simulation of failure in particle composites. Computers $\& 3$ Structures, 44(1-2):459-468, 1992. 
[21] A Suwito, X-C Cai, and Y Xi. Parallel Finite Element Method for Coupled Chloride and Moisture Diffusion in Concrete. Int. J. Numer. Anal. Model., 3(4):481-503, 2006.

[22] L Wang and T Ueda. Mesoscale modeling of water penetration into concrete by capillary absorption. Ocean Engineering, 38(4):519-528, 2011.

[23] Y Xi and Z P Bažant. Modeling Chloride Penetration in Saturated Concrete. J. Mater. Civ. Eng., 11(1):58-66, 1999.

[24] Y Xi, Z P Bažant, L Molina, and M Jennings. Moisture diffusion in cementitious materials: Moisture capacity and diffusivity. Adv. Cem. Based Mater., 1(6):258-266, 1994.

[25] C Yang and S Cho. Approximate migration coefficient of percolated interfacial transition zone by using the accelerated chloride migration test. Cement Concr. Res., 35(2):344-350, 2005.

[26] Z Yuan and J Fish. Toward realization of computational homogenization in practice. Int. J. Numer. Meth. Eng., 73(3):361-380, 2008.

[27] K Zandi Hanjari. Structural Behaviour of Deteriorated Concrete Structures. PhD thesis, Chalmer University of Technology, 2010.

[28] T I Zohdi and P Wriggers. Introduction to Computational Micromechanics. Springer, Berlin, 2005. 